\title{
LA CONSTRUCTION DU SENS POUR LES TERMES MÉTAPHORISÉS EN OSTÉOPATHIE BIODYNAMIQUE À L'AIDE DU CONTEXTE SÉMANTIQUE
}

\section{IRINA KAL,IN,INA}

Université de Lettonie, Lettonie, avec Université Lumière Lyon 2, France

\begin{abstract}
Résumé. La présente étude examine les métaphores terminologiques dans le contexte de la recherche cognitive, précisément leur contribution à la construction du sens et la structuration conceptuelle dans le domaine de l'ostéopathie biodynamique avec des implications possibles sur les questions d'équivalence en traduction. En absence des définitions concrètes pour les termes en ostéopathie biodynamique, nous essayons de les construire à travers le contexte sémantique dans la langue source (anglais). Cela donne un aperçu de la composition du sens (à travers l'inférence sélective) que le terme devrait transmettre et permet de visualiser des paradigmes épistémologiques possibles du domaine en question. Cette information pourrait s'avérer très utile pour les traducteurs qui cherchent à établir un équivalent harmonieux pour les termes (nous examinons les pratiques de traduction en italien et en russe), puisque les métaphores peuvent être profondément ancrées dans la culture de la langue source (Cortès, 2003).
\end{abstract}

Mots-clés : métaphores terminologiques, équivalence, concepts, définition, contexte

\section{INTRODUCTION}

Cet article est une investigation de la notion complexe de métaphore conceptuelle dans le domaine de la terminologie. L'étude propose de formuler d'un modèle définitoire pour les termes en ostéopathie biodynamique à travers l'observation de la formation des relations entre les éléments du sens : ceux qui sont inhérents à l'unité linguistique et ceux qui dépendent du contexte, dans le but de déterminer les constituants sémiques des termes et d'en formuler les définitions.

\section{BASE THÉORIQUE}

Kleiber (2016) distingue trois emplois de la métaphore : celles dites lexicales, impliquant un aspect 'transgressif' dans l'usage d'un mot, les métaphores d'objets 
et les métaphores onomasiologiques, qui permettent de représenter des entités abstraites. C'est cette dernière catégorie qui présente un intérêt particulier pour cette étude.

On suit la définition de la métaphore issue du cadre de la théorie conceptuelle ou cognitive basée principalement sur les travaux de Lakoff et Johnson (1980). Ici, la métaphore est vue comme un mécanisme qui consiste en une projection de la structure d'un domaine d'expérience particulier sur un autre domaine, un outil paradigmatique qui oriente notre pensée, un instrument indispensable qui se situe au cœur de la construction d'une communication efficace. On envisage la métaphore terminologique en tant que métaphore conceptuelle ancrée dans un domaine spécifique d'activité où elle devient l'expression d'un nouveau concept, doté du côté linguistique des caractéristiques particulières.

Même si la présence des métaphores en terminologie a été contestée dans le passé (dans la logique dite Wüsterienne (Trojar, 2007) de la théorie générale de la terminologie qui prétend que les unités terminologiques ne peuvent admettre aucune ambiguïté), aujourd'hui la métaphore jouit d'un statut incontesté parmi les des procédés de formation des unités terminologiques. Comme le remarque Hermans (1989 : 143) 'toute science se fonde sur une opération de métaphorisation, où les glissements de sens, les analogies et l'ambigüité des concepts de base fournissent les hypothèses et guident l'observation'.

La traduction complique l'interprétation des métaphores, puisque, comme le souligne Montuschi, 'même si les différents domaines du savoir et de la pratique sont souvent les mêmes d'un pays à l'autre, les façons de les découper et de les dénommer ne sont pas les mêmes, en vertu de la relative autonomie du langage' (Montuschi, 1993, cité dans Rossi, 2014 : 5).

Afin de mieux saisir le sens des métaphores terminologiques, il paraît nécessaire d'en établir les composantes. D'après Cortès, 'la construction du sens métaphorique présuppose une maitrise totale de la construction du sens compositionnel'. Ce sens, qui est 'un construit énonciatif complexe', nécessite de vastes connaissances de type non seulement lexical, mais aussi encyclopédique. Cela nous donne 'l'accès à l'interdiscours' (Cortès, 2003 : 35).

\section{PROBLÉMATIQUE}

Pour cette étude, on a choisi d'étudier les métaphores dans un domaine spécifique de la science - l'ostéopathie biodynamique. Cette discipline est une branche de la médecine manuelle basée sur le contact physique, originaire des ÉtatsUnis. L'ostéopathie biodynamique (ou traditionnelle) se présente comme une continuation de la lignée directe des fondateurs de la discipline.

Dans notre étude précédente (Kalinina, 2018), on a pu déterminer que 93\% des termes appartenant à cette branche de l'ostéopathie sont des métaphores. Les problèmes de caractère linguistique concernant cette terminologie sont multiples. 
Premièrement, la discipline ne dispose pas de glossaire ni de vocabulaire uniforme pour les termes. Les définitions sont rarement présentes dans les textes et parfois différentes d'un texte à l'autre. La traduction accentue le problème d'ambiguïté, car les traducteurs ne sont pas encore initiés à l'univers sensoriel ostéopathique - la majorité des termes sont traduits par calques ou emprunts directs. Les professeurs d'ostéopathie biodynamique considèrent le langage comme un instrument imparfait pour la transmission de la pensée, et préconisent plutôt le transfert de connaissances par le toucher et d'autres sens. Lors des séminaires, les étudiants apprennent que l'ostéopathie est une tradition 'orale' il existe relativement peu de publications. Cela explique le côté plutôt ésotérique (pour un non-initié) de cette science - d'où la réticence des enseignants à l'idée de voir leurs paroles publiées.

Les métaphores en ostéopathie manquent souvent de transparence. Si l'on considère que la métaphore terminologique doit être 'familière, éculée, transparente', ne pas nécessiter de 'recherche de l'esprit, car son caractère de trope doit échapper à la conscience de l'étudiant afin d'évacuer toute confusion possible' (Oliveira, $2008: 10$ ) - pour l'ostéopathie biodynamique cela n'est pas vrai.

Dans cette discipline, on a affaire à des métaphores vives. $\mathrm{Si}$, par exemple, dans le terme 'inflammation' il reste peu de 'flamme' ou 'feu', dans le terme ostéopathique 'breath of life' (souffle de vie), le mouvement de la respiration est on ne peut plus présent pour le médecin. On se demande, par conséquent, si les métaphores dans ce domaine sont véritablement vouées à une 'évolution obligatoire' vers une métaphore morte (catachrèse) comme le décrivent Bowdle et Gentner (2005: 193-216) qui présente un haut degré de lexicalisation.

Dans la présente étude, on essaie d'effectuer un examen plus approfondi de la terminologie biodynamique. Le but serait d'essayer de clarifier la signification des termes à travers leur contexte afin d'évaluer l'équivalence en traduction. En particulier, notre étude vise à noter l'environnement immédiat des termes décrire le contexte d'usage, identifier les collocations les plus fréquentes, dégager les mots-clés de leur contexte linguistique, et ensuite, en se fondant sur ces données, proposer une définition du terme en question, identifier les domaines sources des métaphores en biodynamique, identifier la cartographie de la métaphore en langue source et examiner son fonctionnement dans les langues cibles. Pour étudier l'inférence sélective, on essaie d'observer la modification sémantique qui advient lors de la métaphorisation - et si cela se produit de la même manière dans différentes langues.

\section{MÉTHODES}

Le corpus de notre étude comprend 60 termes issus de travaux récents (2005-2018). La bibliographie comprend 10 manuels pour le cursus complet d'entrainement dans l'art de l'ostéopathie biodynamique, 3 manuels plus spécifiques en pédiatrie et un livre intitulé 'Odyssée ostéopathie', écrit et publié par l'enseignant principal de cette approche, le docteur James Jealous. 
Pour effectuer une analyse approfondie du contexte, on a noté chaque terme et son environnement immédiat dans plusieurs sources en anglais. Ensuite, on a identifié les mots-clés. À partir de ces données, une définition courte et une autre plus descriptive ont été dégagées. Ensuite, on a identifié le sens du mot (ou du syntagme) du domaine source dans chaque langue pour en comparer les traits avec le sens du mot (ou du syntagme) dans le domaine cible. Les données ainsi obtenues ont été analysées d'une manière qualitative et quantitative.

Vu que la métaphore s'appuie sur un 'mapping' (cartographie), on a d'abord identifié les domaines sources pour les termes en ostéopathie biodynamique : ils varient de la langue générale à la terminologie des autres domaines. Tandis que $76 \%$ des termes métaphorisés ont un seul domaine source, par exemple, ignition (automobile), les 24\% restants ont plus d'un domaine source, par exemple : fluid drive (substances et phénomènes physiques).

Comme la cartographie est un processus partiel où, selon Oliveira (2005:6), 'seule une partie du réseau complexe de gestalts expérientielles qui constituent la structure du domaine source sera projetée sur le domaine cible', lors du passage advient une modification sémantique. Les termes qu'on a pu observer dans le cadre de cette recherche manifestent une réduction où une partie seulement du sémème est transférée avec, parfois, l'ajout d'autres sèmes.

$\mathrm{Du}$ point de vue de l'inférence sélective, les termes-métaphores de notre corpus appartiennent tous à la catégorie des 'métaphores interactives'. Comme l'explique Oliveira (2005: 6), cette métaphore 'compare de manière implicite et son but est de surprendre l'esprit et, par là, d'inciter le scientifique à rechercher les similitudes existantes entre les deux domaines. À la suite de ce conflit cognitif, le spécialiste est amené à considérer l'objet de la métaphore dans une perspective différente, ce qui le prédispose à effectuer un changement conceptuel'.

L'observation des unités du corpus nous a permis de constater que, du point de vue sémiotique (d'après Prandi 2012, cité par Rossi, 2014 : 715), les métaphores terminologiques dans le domaine de l'ostéopathie biodynamique constituent toutes un renvoi cognitif à un réseau lexical partagé (isotopie), fonctionnel pour la création des termes. Cette disposition nous oblige à défier la cohérence du discours ordinaire afin de créer de nouvelles conceptualisations. Ainsi, ce sont des structures plus complexes que des catachrèses à la base d'une analogie formelle.

Le cadre de cette communication ne nous permet pas de donner beaucoup d'exemples car chacun nécessite une longue explication, mais on présentera les résultats essentiels.

\section{RÉSULTATS}

À la suite de notre analyse, on a noté la modification sémantique qui advient par la suppression (comme dans le cas de 'CV4, compression of the fourth ventricle' qui n'est pas une compression, mais une procédure par laquelle les forces du 
'fluide dans le fluide' sont amenées au centre en créant le neutre, à travers lequel les forces thérapeutiques s'expriment), mais aussi par l'ajout de certains traits. Le concept du terme 'fire', par exemple, suppose non seulement la possibilité de brûler le vieux, mais aussi de construire du nouveau, comme dans 'Fire brings shape and boundary into the function it creates' (manuel phase 8).

Certaines métaphores sont basées sur des images très complexes où une métaphore en cache d'autres, de manière implicite. Le terme 'neutral' (point mort) provient initialement du domaine de l'automobile. La définition issue du contexte se présente comme 'une qualité de paix, quiétude et repos pour le corps et l'esprit du patient, ainsi que de l'ostéopathe, qui n'est ni passif ni actif, mais se trouve dans un état réceptif aux forces de la guérison'. Mais, ce qui est implicite, c'est que le 'neutre' se base sur l'image d'une voiture dont le moteur est en marche, mais qui reste sur place, l'ostéopathe étant le passager et 'the Master' (le Maître) étant au volant. Leur voyage est un mystère, et leur direction obéit à la volonté divine (manuel sur la Pédiatrie N3).

Plusieurs problèmes peuvent survenir lors du transfert des termes en d'autres langues. La conceptualisation passe largement par la culture de la langue source (Temmerman, 2000). Les liens sémantiques se voient parfois modifiés considérablement. C'est en partie ainsi que la langue de formation terminologique arrive à dominer dans un secteur donné. Un autre point à considérer dans le cadre de la terminologie ostéopathique est l'idiolecte des créateurs des termes dans cette discipline. Chez les ostéopathes, la connaissance passe par diverses voies, et celle de la parole n'est que l'une d'entre elles. Ainsi, on pourrait supposer que beaucoup d'information reste implicite, même si elle est partagée dans la plupart des cas. Or, c'est l'implicite qui devrait être mobilisé lors du transfert, comme le note Rossi (2014).

La traduction des termes ou 'création secondaire' (Sager, 1990 : 82) est un processus où un nouveau terme est créé à partir d'un concept déjà compris. Le problème est que le plus souvent, ce processus de création est effectué par les spécialistes eux-mêmes, de manière assez désordonnée. Les traducteurs et interprètes, d'un autre côté, bien que spécialistes au niveau linguistique, éprouvent une certaine réticence à s'impliquer dans la création des termes dont les concepts leur restent, la plupart du temps, assez opaques, faute de définitions.

Parmi les procédés de formation secondaire des termes proposés par Rossi (2014), on observe que :

- Le mécanisme métaphorique de base est partagé dans $56 \%$ de cas en russe et $63 \%$ de cas en italien. Exemple : Breath of Life - Dyhanie Zhizni (respiration de vie) : la métaphore est fondée sur l'expression équivalente issue de la Bible.

- L'adaptation du champ lexical à un autre contexte advient dans $30 \%$ des cas en russe et $22 \%$ des cas en italien. Breath of Life en italien devient Soffio vitale. Dans la Genèse, le terme analogue qui apparaît est 'alito di vita'. On a donc préféré utiliser un terme plus proche du domaine du 
pranayama. Ce type d'adaptation est fréquent en italien - la forme du terme est rendue par calque, mais le sens est légèrement modifié car, comme dans ce cas, il n'y plus l'association directe avec Dieu qui insuffle cette respiration en l'homme. En pranayama, ce souffle est indépendant.

- La perte de la force heuristique de la métaphore advient quand elle se trouve éliminée dans la langue cible, le terme étant alors traduit par un emprunt intégral ( $13 \%$ des cas en russe et $13 \%$ des cas en italien). Cela fait courir au terme le risque de devenir opaque pour les locuteurs, notamment ceux qui n'ont pas de connaissances en anglais. Exemple : 'stillness' qui est rendu par 'stilnes' en russe, une transcription directe.

Quant aux procédés de traduction pour la partie formelle du terme, les résultats sont presque les mêmes dans les deux langues cibles: le calque (ou équivalent formel) est employé dans $61 \%$ des cas en russe et $68 \%$ en italien, un terme analogue qui ne copie pas la structure du terme source dans $22 \%$ des cas en russe et $9 \%$ en italien, l'emprunt intégral dans $17 \%$ des cas en russe et $23 \%$ en italien. L'italien s'avère légèrement plus perméable à la langue source (anglais) que le russe.

La traduction par calque avec une certaine modification du champ sémantique du terme peut amener à des déformations conceptuelles. Dans le cas de 'fluid' (une substance de base avec un haut potentiel de transformation) - 'fluid', le mot en russe provient d'un contexte complètement différent (celui du spiritisme), il porte ainsi des associations différentes du terme source. Ainsi, il est difficile d'affirmer que la base métaphorique est entièrement préservée. En même temps, les praticiens s'opposent à l'usage du mot 'zhidkost' (liquide), qui leur parait inadéquat pour décrire la réalité en question.

Pourtant, la préférence pour le calque comme l'affirme Shuttleworth (2017 : 19) peut être expliquée par une 'attraction gravitationnelle' (gravitational pull) puissante que la langue source exerce sur les traducteurs, qui se montrent réticents à rechercher des analogues dans la langue cible.

Un autre phénomène auquel on a eu affaire pendant le travail est une certaine absence de rigueur terminologique dans la langue source: la capitalisation, par exemple, détermine le terme, mais elle est parfois mal employée. 'Tide' (la présence vivante de Respiration de Vie qui passe à travers toute chose en apportant la transmutation) et 'tide' (la force électromagnétique dans le fluide) sont deux concepts distincts où la capitalisation détermine le sens, mais parfois l'un est écrit à la place de l'autre. Seul le contexte nous permet d'établir duquel il s'agit.

Un problème encore plus grave est que certains termes sont parfois employés comme synonymes et parfois distingués (c'est le cas de 'Tide', 'Breath of Life' et 'Primary Respiration'). L'enseignant peut employer les termes de façon imprévisible pour le traducteur, arguant du fait que les participants savent très bien de quoi il s'agit, puisqu'ils perçoivent l'information par d'autres voies que celle de la parole. 


\section{CONCLUSION}

Le travail effectué et l'observation des unités du corpus nous ont permis de conclure que la création secondaire des termes en langues cibles passe par le mécanisme métaphorique de base partagé dans la majorité des cas, mais aussi à travers l'adaptation du champ lexical et finalement dans quelques cas avec une perte de la force heuristique de la métaphore. La terminologie en ostéopathie biodynamique posant certains problèmes au niveau de la systématisation, de la compréhension et de la traduction, la construction de la définition à partir du contexte linguistique aide considérablement à la compréhension du terme et pourrait servir à l'évaluation et à l'établissement des équivalents dans les langues cibles.

Cette étude va être poursuivie pour inclure d'autres termes en biodynamique et d'autres langues-cible.

\section{BIBLIOGRAPHIE}

Bowdle, B. F. et Gentner, D. (2005) The career of metaphor. Psychological Review, 112 (1) : 193-216.

Cortès, C. (2003) La métaphore: du discours général aux discours spécialisés. Paris, France : Université Paris 7 Denis Diderot.

Hermans, A. (1989) La définition des termes scientifiques. Meta : Journal des traducteurs / Meta : Translators' Journal, 34 (3) : 529-532.

Kalinina, I. (2018) Translating metaphors in osteopathic terminology: the subtlety challenge. Conference proceedings 'XXVIII Scientific Readings', 68-74.

Kleiber, G. (2016). Du triple sens de métaphore. Langue Française, 189 : 15-34.

Lakoff, G. (1993) The contemporary theory of metaphor. In A. Ortony (ed.) Metaphor and Thought (pp. 202-251). New York, NY, US: Cambridge University Press.

Lakoff, G. et Johnson, M. (1980) Metaphors we live by. Chicago and London: University of Chicago Press.

Montuschi, E. (1993) Le metafore scientifiche. Milano : Franco Angeli.

Oliveira, I. (2005) La métaphore terminologique sous un angle cognitif. Meta: Journal des traducteurs / Meta: Translators' Journal, 50 (4). Disponible à https://www.erudit.org/fr/ revues/meta/2005-v50-n4-meta1024/019923ar/ [Consulté le 2 février 2019].

Oliveira, I. (2008) Métaphore terminologique et stratégie pédagogique. Analele Universității din București. Limba și literatura română, LVII (pp. 1220-1271). Disponible à http:// www.diacronia.ro/en/indexing/details/A2423 [Consulté le 4 février 2019].

Rossi, M. (2014) Métaphores terminologiques : fonctions et statut dans les langues de spécialité. SHS Web of Conferences, 8 : 713-724.

Sager,J. C. (1990) A practical course in terminology processing. Amsterdam, États-Unis d’Amérique : John Benjamins Publishing.

Shuttleworth, M. (2017) Studying scientific metaphor in translation: an inquiry into cross-lingual translation practices. New York, États-Unis d'Amérique, Royaume-Uni de Grande-Bretagne et d'Irlande du Nord : Routledge.

Temmerman, R. (2000) Towards new ways of terminology description: the sociocognitive-approach. Amsterdam : John Benjamins Publishing Company.

Trojar, M. (2007) Wüster's View of Terminology. Slovenski jezik - Slovene Linguistic Studies, $11: 55-85$. 


\title{
MEANING CONSTRUCTION FOR METAPHORIZED TERMS IN BIODYNAMIC OSTEOPATHIC TERMINOLOGY WITH THE HELP OF SEMANTIC CONTEXT
}

\begin{abstract}
The proposed study examines terminological metaphors in the context of cognitive research, namely their contribution to meaning construction and concept structuring in the field of biodynamic osteopathy with possible implications for translation equivalence issues. In the absence of clear definitions for the terms used in biodynamics of osteopathy, we attempt to build them via the semantic context in the source language (English). This provides an insight into the composition of meaning (by selective inference) that the term is supposed to relay and gives an opportunity to visualize possible epistemological paradigms of the relevant domain. Such information may prove valuable for translators attempting to select a congruent equivalent for terms (we examine translation practices in Italian and Russian), as metaphors can be deeply anchored in the source-language culture (Cortès, 2003).
\end{abstract}

Key words: terminological metaphor, equivalence, concept, definition, context

Irina Kalıinina (doctorante en cotutelle en linguistique avec l'Université de Lettonie et l'Université Lyon 2) travaille sur un projet à l'Université de Lettonie et enseigne à l'Université de Milan en Italie. Ses intérêts de recherche incluent la traduction et la recherche terminologique en anglais, français, italien, letton et russe.Adresse e-mail : irina.kalinina8118@gmail.com 